\title{
Globe
}

Revue internationale d'études québécoises

\section{Authenticités québécoises. Le Québec et la fragmentation contemporaine de l'identité}

\section{Jocelyn Maclure}

Volume 1, numéro 1, 1998

Raisons communes

URI : https://id.erudit.org/iderudit/1000099ar

DOI : https://doi.org/10.7202/1000099ar

Aller au sommaire du numéro

Éditeur(s)

Globe, Revue internationale d'études québécoises

ISSN

1481-5869 (imprimé)

1923-8231 (numérique)

Découvrir la revue

Citer cet article

Maclure, J. (1998). Authenticités québécoises. Le Québec et la fragmentation contemporaine de l'identité. Globe, 1(1), 9-35. https://doi.org/10.7202/1000099ar
Résumé de l'article

Une partie des nationalistes québécois continuent de penser l'identite québécoise dans les termes d'une éthique d'authenticité qui définit de façon substantive ce qu'est la québécitude. En réaction contre cet incorporation de l'identité québécoise dans des catégories hermétiques, un courant antinationaliste/cosmopolitique s'est développé au Québec. Or, une réflexion sur la fragmentation de l'identité moderne et sur le sentiment de dépaysement qui habite le sujet contemporain empêche toute tentative de fixation identitaire (qu'elle soit nationaliste ou cosmopolitique). C'est pourquoi l'identité québécoise doit être pensée dans les termes d'une éthique de créativité et d'une authenticité hybride qui permet à la fois l'enracinement et l'arrachement.
Tous droits réservés @ Globe, Revue internationale d’études québécoises, 1998

The de

Ce document est protégé par la loi sur le droit d'auteur. L'utilisation des services d'Érudit (y compris la reproduction) est assujettie à sa politique d'utilisation que vous pouvez consulter en ligne.

https://apropos.erudit.org/fr/usagers/politique-dutilisation/ 


\title{
Authenticités québécoises. Le Québec et la fragmentation contemporaine de l'identité 1
}

\author{
Jocelyn Maclure \\ Université de Victoria
}

La problématique de l'identité se veut aujourd'hui l'un des thèmes de recherche dominants tant en philosophie sociale et politique qu'en politique comparée et en théorie littéraire. Certains diront que cette réflexion n'est en fait que fabulation et fantasme créés par des gens de lettres et d'esprit qui tentent de justifier leur pertinence en discourant sur un enjeu théorique artificiel, autonome et disjoint de la raison pratique. D'autres, parmi lesquels je me range, soutiennent que le questionnement identitaire est incontournable et insoluble, puisqu'il est intrinsèquement lié à une modernité aux tribulations incessantes. L'époque contemporaine, marquée entre autres choses par une compression inégalée du temps et de l'espace, exige une réarticulation et une reconceptualisation des «frontières de l'identités. Or, bien que ce projet théorique soit indéniablement déjà amorcé ${ }^{2}$, on peut avancer que le débat sur

1 Je tiens à remercier James Tully et Marie-Josée Deblois pour leur soutien.

2 Voir par exemple : Mikhaël Elbaz, Andrée Fortin et Guy Laforest [éd.], Les Frontières de l'identité. Modernité et postmodernisme au Québec, Sainte-Foy et Paris, Presses de l'université Laval et L'Harmattan, 1996; Marcos Ancelovici et Francis Dupuis-Déri, L'Archipel identitaire; Recueil d'entretiens sur l'identité culturelle, Montréal, Boréal, 1997; Dimitrios Karmis, «nterpréter l'identité québécoise» dans 
l'identité contemporaine est encore largement dominé au Québec par la confrontation traditionnelle entre les shérauts de l'authenticité» et les "pourfendeurs séculaires du nationalisme».

La plupart des recherches contemporaines sur lidentité, qu'elles soient menées par des auteurs qualifiés de «postmodernistes» ou non, a révélé une complexité, une polyphonie et une fluidité dont s'acquittent difficilement les ontologies libérale / cosmopolitique et nationaliste / "communautarienne» traditionnelles. Par conséquent, l'objectif de ce texte - qui se veut davantage une brèche ouverte dans un univers théorique en pleine émergence qu'une tentative de synthèse - sera d'abord de montrer l'obsolescence des conceptions dominantes de l'identité québécoise, afin d'en proposer ensuite une interprétation. Après avoir établi que toute tentative de fixation identitaire, nationaliste ou cosmopolitique, est contraire à une identité contemporaine conçue comme projet narratif, on tentera de montrer que c'est dans les termes d'une éthique de créativité, d'inspiration foucaldienne, que s'exprime et se comprend le mieux l'identité québécoise. Finalement, pour conclure ces «prolégomènes à une interprétation future de lidentité québécoises, une reconceptualisation de l'identité québécoise qui tient pleinement compte de la fragmentation identitaire contemporaine sera esquissée.

\section{Le nationalisme québécois et la recherche d'une authenticité perdue}

Malgré ce qu'en pensent ses pourfendeurs, le nationalisme québécois est loin d'être un courant homogène et monolithique.

Alain G. Gagnon [éd.], Québec. État et sociêté, Montréal, Québec/Amérique, 1994, pp. 305-327. 
Des indépendantistes, vaille que vaille, aux partisans d'un fédéralisme canadien multinational, des souverainistes "par défaut» aux partisans de la société distincte, des «intégrationnistes» aux «assimilationnistes», le nationalisme québécois ne s'exprime pas d'une voix commune. Comme le notent Dimitrios Karmis et Joseph Carens, la variante (intégrationniste" du nationalisme québécois, qui accueille l'immigrant sans lui demander d'abandonner son identité mémorielle forme aujourd'hui la tendance qui est à la base de l'élaboration des politiques officielles de l'État québécois ${ }^{3}$. Malgré cela, il reste bon nombre de nationalistes au Québec professeurs, intellectuels, artistes, politiciens et autres citoyens qui considèrent qu'il existe une façon authentique d'être Québécois(e) et qu'il est possible de définir substantivement les éléments constitutifs de la québécitude. Par exemple, certains considèrent que le sort de l'identité québécoise s'est joué sur les Plaines d'Abraham et que, depuis ce temps, les Québécois(es) refoulent sans cesse le traumatisme originel que fut la Conquête ${ }^{4}$. Selon cette hypothèse, le Québécois contemporain, qu'il le sache ou non, doit composer avec un $\xi a$ qui puise son énergie libidinale dans une défaite historique datant du XVIIIe siècle. L'intériorisation d'un passé colonial ferait alors partie intégrante d'une identité québécoise définie de façon substantive. Pour d'autres, la québécitude est une version améliorée ou diluée (selon que l'on s'adresse à Maurice Duplessis ou à Jean Larose) de la francité. Enfin, d'autres affirment au

3 Dimitrios Karmis, "Interpréter l'identité québécoise», loc. cit., pp. 318-319. Joseph H. Carens, «Immigration, Political Community, and the Transformation of Identity : Québec's Immigration Policies in Critical Perspective», Is Québec Nationalism Just? Perspective from Anglophone Canada, McGill-Queen's University Press, 1995.

4 Pour une critique efficace de ce genre d'argument, voir le commentaire de Dimitrios Karmis au sujet du livre de Christian Dufour, Le Défi québécois dans "Interpréter l'identité québécoise», loc. cit., pp. 307-308. 
contraire que le vrai Québécois est un Américain qui s'exprime en français. Or, en chosifiant l'identité québécoise de la sorte, le risque est grand de faire du Québécois une espèce en voie de disparition! Indubitablement, si l'on peut définir l'essence de l'identité québécoise, l'on peut aussi déterminer qui est un Québécois authentique et qui ne l'est pas. C'est ainsi par exemple que Jacques Parizeau, donnant dans l'expatriation identitaire, considère que l'indépendantisme est au cœur de l'authenticité québécoise :

Se sentir québécois, cela vient petit à petit. J'ai moimême été fédéraliste jusqu'à la fin de la trentaine. J'ai changé d'option quand je me suis rendu compte que dressés l'un contre l'autre, le Québec et le Canada se neutralisent, n'arrivent plus à bouger, s'enfoncent dans des conflits souvent dérisoires. Je n'en veux pas à ceux qui ont décidé d'être canadiens. Moi j'ai choisi, comme bien d'autres, d'être québécois 5 .

Cette «version québécoise de l'identité républicaine française», pour reprendre l'expression de Karmis, où il n'y a qu'une seule bonne façon d'adhérer au tout, manifeste sa présence dans toutes les sphères de la société québécoise. À titre d'exemple, l'arpenteur-écrivain décrit par Monique LaRue, qui navigue laborieusement à travers les vagues de l'identité contemporaine, tente lui aussi de figer lidentité québécoise dans des catégories hermétiques et authentiques. Se basant sur «l'héroïque antériorité» des écrivains québécois "de souche», l'arpenteur s'en prend à cette nouvelle génération d'écrivains immigrants qui fait éclater, tant au niveau thématique que stylistique, les frontières de la littérature québécoise. Ces écrivains, attachés à leurs mémoires, ne poursuivent pas la «recherche d'identité» et ne

5 Jacques Parizeau, «Lettre ouverte aux souverainistes», Le Devoir, 19 décembre 1996, p. A-7. 
s'approprient pas le «réseau de références», la «dynamique intertextuelle» et «'imaginaire» propres à la littérature québécoise $^{6}$. En bref, les écrivains immigrants, aux dires de l'arpenteur 'décrit par Monique LaRue, constituent une menace pour la «singularité» - voire l'authenticité - de la littérature québécoise. Une fois réifiée, la littérature peut, elle aussi, servir d'étalon pour mesuret le degré d'(in)authenticité des écrivains québécois venus d'ailleurs.

La société québécoise apparaît donc encore traversée par un courant "substantialiste» qui embrigade la québécitude au sein d'une clôture identitaire hermétique. L'identité québécoise est une question fermée à laquelle l'histoire a déjà trouvé réponse. $A$ contrario de ce que laissent croire ses plus fervents critiques, cette faction importante du nationalisme québécois n'est pas fondée - dans sa majorité - sur des critères ethniques qui feraient du sang et de la "souche» la base de l'identité québécoise, mais plutôt sur une éthique d'authenticité construite socialement et culturellement, et à laquelle le Québécois de toute origine doit adhérer afin d'éviter la facticité.

\section{L'idéal cosmopolitique et les pourfendeurs du nationalisme québécois}

Les critiques les plus sérieuses et les plus véhémentes du «républicanisme» québécois sont souvent portées au nom d'un idéal cosmopolitique qui disqualifie de facto toute tentative d'essentialiser et de réifier certains éléments de l'identité québécoise. Par exemple, selon Régine Robin, au Québec «'identité est une essence, donnée a priori, elle émane de ce caractère français des habitants à l'origine et de leur langue.

6 Monique LaRue, L'Arpenteur et le navigateur, Montréal, Fides, coll. "CÉTUQ", 1996, p. 8. 
Langue et identité coïncident, de même que langue, identité et culture ${ }^{7 . »}$ Or, toute cette coincidence, qui forme une "glu identitaire», ne peut être présumée que chez ceux qui possèdent une langue, une identité et une culture communes et qui partagent, pour reprendre l'expression de Robin, le même «roman mémorieb». En d'autres termes, lidentité québécoise contemporaine n'est accessible qu'à la communauté imaginée canadienne-française, selon son appellation pré-Révolution tranquille. Le nationalisme québécois, dévêtu de ses oripeaux civiques et territoriaux, demeure un projet qui s'adresserait aux Québécois(es) «de souche» et qui engendrerait, par le fait même, de l'exclusion.

De ce diagnostic qui correspond en partie à la réalité, les pourfendeurs contemporains du nationalisme québécois tirent habituellement des conclusions politiques d'inspirations vaguement kantiennes contraires à la volonté d'une majorité de Québécois de penser le Québec comme une communauté politique distincte et autonome. En effet, la transition entre l'antinationalisme d'un côté, et le libéralisme procédural doublé d'un certain universalisme de l'autre, va de soi pour ceux qui, comme Robin, Derriennic ou Angenot, ont pris le relais des Citélibristes d'antan. Plurielles et hybrides, les sociétés contemporaines doivent composer avec une multiplicité de visions de la vie bonne. Par conséquent, le rôle du politique est de distribuer aux personnes des droits individuels et de s'assurer qu'aucun sous-groupe, au sein de la société, ne puisse imposer politiquement sa vision du Bien. Comme le rappelle Jean-Pierre Derriennic, «les lois justes ne sont pas celles qui ont été faites sur

7 Régine Robin, «Défaire les identités fétiches», La Question identitaire au Canada francophone; Récits, parcours, enjeux, hors lieu, Sainte-Foy, Presses de l'université Laval, 1994, p. 216. Dans la même veine, voir également son article "L'impossible Québec pluriel : la fascination de la "souche"》, Les Frontières de l'identité, op. cit., pp. 295-310. 
mesure pour une personne ou un groupe ${ }^{8} . »$ La légitimité de politiques "communautariennes», conçues par exemple pour la majorité francophone du Québec, devient ainsi problématique. Qui plus est, puisque les identifications et allégeances nationales créent uniquement de la différenciation et de l'exclusion, les auteurs antinationalistes ne peuvent qu'en arriver à plaider pour un idéal universaliste et cosmopolitique. «À la problématique de la différence, il faut, je crois, opposer fermement dans un monde hostile qui se ghettoïse une problématique de l'altérité, à penser dans le cadre d'un nouvel universalismes, conclut d'ailleurs Robin à cet effet?. Même Monique LaRue, comme bien d'autres défenseurs de l'hybridité et du métissage qui ne sont pas antinationalistes pour autant, semble parfois penser que la meilleure façon de libérer le Québec de sa vision "d'arpenteur-géomètre» serait peut-être de penser l'identité québécoise dans une perspective universaliste ${ }^{10}$.

Or, on avancera dans les prochaines parties que, malgré leur objectif avoué (et légitime) de mettre un terme à une certaine suffocation identitaire qui règne au Québec, les antinationalistes enferment aussi la québécitude dans des catégories identitaires pré-établies et échouent eux aussi à la tâche de penser l'identité contemporaine dans toute sa complexité.

8 Jean-Pierre Derriennic, Nationalisme et démocratie. Réflexion sur les illusions des indépendantistes québécois, Montréal, Boréal, 1995, p. 38.

9 Régine Robin, «Défaire les identités fétiches», loc. cit., p. 238.

10 Voir par exemple des allusions telles : «heureux les philosophes, me disais-je, qui ne connaissent pas de frontières nationales» ou ímagine ce que serait la littérature québécoise si elle devenait simplement littérature», Monique LaRue, L'Arpenteur et de la navigateur, op. cit., pp. 20 et 28. 


\section{Interpréter l'identité contemporaine}

Comme le rappelle Charles Taylor, avec la période modeme est apparue une nouvelle conception de l'identité personnelle ${ }^{11}$. Envisagée comme une "destruction créatrices, la modemité a sonné le glas des sociétés holistes et stratifiées, où l'identité dépendait quasi-exclusivement de la position occupée dans la hiérarchie sociale, et a inauguré une ère où le sujet doit prendre sur ses épaules l'élaboration de sa propre identité. En d'autres termes, avec l'émergence de la modernité, l'identité cesse d'être une donnée a priori que l'individu doit assumer plutôt que de façonner ${ }^{12}$. L'identité devient ainsi une narration, un projet biographique que le sujet réflexif doit composer en évoluant au sein d'un envitonnement en constante transformation. $\grave{A}$ cet effet, Michel Freitag considère que «la synthèse de cette réflexivité, par laquelle le sujet (ije») s'érige au-dessus d'une simple subjectivité diffuse et immédiatement actuelle pour devenir précisément «un» sujet doté d'une certaine permanence pour lui-même et pour autrui, c'est lidentité ${ }^{13 . » ~ D o n c, ~ t e l ~ q u e ~ l e ~}$

11 Charles Taylor, Multiculturalisme. Différence et démocratie, Paris, Aubier, 1994, p. 44.

12 Il ne s'agit pas ici de simplifier indûment ou de figer l'identité prémoderne, mais plutôt d'exhiber les conséquences du passage d'une période dominée par le maintien de l'ordre et de la stabilité à une période caractérisée par le mouvement et la rupture sur la formation de l'identité individuelle.

13 Michel Freitag, «L'identité, l'altérité et le politique. Essai exploratoire de reconstruction conceptuelle-historique», Société, Québec, no 9 (hiver 1992), p. 1. Il est à noter que, si la définition conceptuelle de l'identité offerte par Freitag m'apparaît satisfaisante, je ne peux partager l'une de ses conclusions voulant que cette notion soit maintenant éclatée et impossible à recomposer dans le contexte d'une transition vers la postmodernité. La justification de cette opposition, qui demanderait un examen approfondi des conditions 
suggère Mikhaël Elbaz, «l'identité peut être saisie comme une fiction persuasive et une opération narrative plutôt qu'une condition objective ou primordiale. $\rangle^{14}$ Or, malgré le caractère fictif et narratif de la construction identitaire modeme, l'identité n'est pas pour autant une illusion ou un fantasme de l'esprit dont on pourrait bien se passer, comme l'ont laissé entendre certains fossoyeurs de l'idée de sujet. À l'inverse, pris au cœur d'une modernité autodestructrice et perpétuellement mouvante, le sujet contemporain a besoin d'une identité à laquelle il peut se référer afin d'orienter sa praxis. Le sujet puise dans son identité les horizons qui lui permettent de maintenir une certaine cohérence dans l'écriture de sa propre biographie :

Ce n'est pas facile à définir, mais on pourrait dire que mon identité définit en quelque sorte l'horizon de mon univers moral. À partir de mon identité, je sais ce qui compte vraiment pour moi et ce qui est de moindre importance. Je sais ce qui me touche profondément et ce qui possède moins de signification. $\mathrm{Ce}$ qui est proprement terrifiant dans la crise d'identité, c'est le fait d'avoir perdu de tels repères ${ }^{15}$.

En résumé, en plus d'être un projet narratif, lidentité procure au sujet, pour travestir un concept utilisé par Giddens,

de possibilité de l'autonomie du sujet contemporain, ne pourra malheureusement pas être effectuée dans le cadre du présent essai. 14 Mikhaël Elbaz, «Introduction», Les Frontières de l'identité, loc. cit., p. 8. Le dernier roman de Milan Kundera est une bonne représentation du caractère fictif de l'identité, où la frontière entre la rêverie et la réalité est ténue et confuse. Milan Kundera, L'Identité, Paris, Gallimard, 1997.

15 Charles Taylor, «Les sources de l'identité moderne», Les Frontières de l'identité, loc. cit., p. 348. 
la "sécurité ontologique»" dont il à besoin pour évoluer dans l'ère de l'incertitude. Toutefois, prétendre que l'identité aide le sujet à répondre à la question "qui suis-je hે n'équivaut pas à affirmer que l'identité est une substance fixe, statique et toujours identique à elle-même. Au contraire, lidentité est une forme instable, floue et dissoluble. Comme le suggère pertinemment Michel Foucault, «les identités se définissent par des trajectoires» et non comme des réalités définies une fois pour toutes ${ }^{17}$. Ce diagnostic est encore plus vrai à l'heure du post-keynesianisme, où les réseaux d'appartenance et d'affiliation du sujet fluctuent et se recomposent selon une dynamique local / global sans précédent ${ }^{18}$. L'identification nationale ébranlée - et non pas effacée - par la prolifération des échanges transnationaux (économiques, culturels, politiques, etc.), l'individu se retrouve confronté à une multiplicité de sources, de filières identificatrices qu'il doit prendre en considération dans la difficile élaboration de son identité. Puisant à la fois dans sa mémoire et dans son imagination, dans ses communautés physiques et virtuelles, le soi contemporain doit assumer la responsabilité de créer sa propre idiosyncrasie identitaire. Les références globales et exhaustives se raréfient, le sujet s'inspire d'une pluralité de référents identitaires partiels, jamais tout à fait adéquats, mais jamais complétement impertinents. D'ailleurs, aux dires du théoricien (postcolonialiste) Homi Bhabha, le sentiment de vertige ou d'étourdissement vécu par le contemporain peut s'expliquer en partie par le fait que nous vivons présentement une période de transition qui produit des figures identitaires inédites :

16 Anthony Giddens, Modernity and Self-Identity; Self and Society in the Late Modern Age, California, Stanford University Press, 1991, p. 38. Voir aussi Ronald D. Laing, Le Moi divisé, Paris, Pluriel, 1979, p. 43. 17 Michel Foucault, «Pour une morale de l'inconforb), Dits et écrits, vol. III, Paris, Gallimard, 1994, p. 786.

18 Voir Jocelyn Létourneau, Les Années sans guide; Le Canada à l'ère de l'économie migrante, Montréal, Boréal, 1996, p. 17. 
[...] in the fin de siècle, we find ourselves in the moment of transit where space and time cross to produce complex figures of difference and identity, past and present, inside and outside, inclusion and exclusion ${ }^{19}$.

Pour les fins de cet essai, la fragmentation de l'identité contemporaine proclamée à l'unisson dans certains milieux intellectuels signifie tout simplement ceci : maintenant plus que jamais, le sujet doit prendre en considération une multiplicité de filières identificatrices dans l'écriture de son récit identitaire. Il est de plus en plus difficile aujourd'hui, même si cela l'a peut-être toujours été, de résumer l'identité d'une personne à une allégeance principale et englobante. Que l'on fasse référence à l'origine, la nationalité, la foi, l'orientation sexuelle, la profession, la sociabilité, le mode de vie, l'engagement politique, l'état civil, ou au genre, il est impossible de capter la complexité du sujet contemporain en n'embrassant qu'un seul de ses fragments de $v^{20} e^{20}$ L'identité est un site problématique et contesté d'élaboration personnelle. La même affirmation s'applique à l'univers culturel auquel appartient l'individu. En tant qu'agent culturel, le sujet chemine au sein de cultures qui ne sont pas des codes immuables, mais bien des processus vivants et évolutifs. Comme le rappelle l'anthropologue James Clifford, une culture est une umultiple authored invention, un «shifting paradox» et une

19 Homi K. Bhabha, The Location of culture, London and New York, Routledge, 1994, p. 1.

20 C'est pourquoi, par exemple, certains homosexuels et immigrants refusent la ghetthoisation et affirment la polyphonie de leur identité, et certaines femmes autochtones réclament le droit d'être à la fois nationalistes et féministes libérales. Voir par exemple : Xavier de Sambourcy, «Désolé, je ne suis pas "fier" d'être gai», Le Devoir, 14 août 1998, p. A.-11. Neil Bissoondath, Le Marché aux illusions; La méprise du multiculturalisme, Montréal, Boréal, 1995. 
«ōngoing translation ${ }^{21}$. Les communautés d'appartenance ne sont pas ces sites homogènes décrits par les penseurs "communautariens» où l'individu doit "découvrin" son identité, mais bien des «zones de contact) où les cultures sont construites et reconstruites selon des dynamiques en constantes fluctuations. En d'autres termes, les cultures sont des «étranges multiplicités $)^{22}$.

"L'homme dépaysé» de Tzvetan Todorov apparaît ainsi comme une figure appropriée pour représenter le sujet contemporain. La transculturation dont parle Todorov ne se limite pas à l'individu qui acquiert une nouvelle identité culturelle sans dépraver pour autant l'ancienne, mais atteint également le «national» qui évolue au sein de communautés physiques et imaginaires qui transcendent les frontières de sa nation $^{23}$. Être dépaysé, cela ne signifie pas (nécessairement) être apatride en son propre pays. Cela signifie plutôt que la fragmentation des identités et des communautés fait en sorte que la coïncidence parfaite entre la partie et le tout, entre l'individu et le groupe est virtuellement impossible. Le sujet contemporain est dépaysé, puisque perpétuellement étonné par la complexité de ses congénères, de sa communauté, de sa nation et de sa propre identité. À la manière de Todorov, Alain Touraine propose pour sa part qu'il faut choisir la figure de l'émigré, ce «voyageur rempli de mémoire autant que de projets et qui se découvre et se construit lui-même dans cet effort de chaque jour pour nouer le passé à l'avenir, l'héritage culturel à l'insertion professionnelle et sociale», comme porte-étendard de

21 James Clifford, Routes. Travel and Translation in the Late Twentieth Century, Cambridge and London, Harvard University Press, 1997, p. 24.

22 Voir James Tully, Strange multiplicity, Constitutionalism in an Age of Diversity, Cambridge University Press, 1995.

23 Tzvetan Todorov, L'Homme dépaysé, Paris, Seuil, 1996, p. 23. 
l'époque actuelle ${ }^{24}$. Penser la fragmentation de l'identité contemporaine n'équivaut donc pas à composer une ode au mouvement et à l'acculturation. Il s'agit plutôt de montrer que, même pour la personne qui choisit (plus ou moins librement) la staticité et dont lidentité semble aisément décernable, il y a dans ses souvenirs, sa vision du futur, ses origines et ses communautés de sentiment, davantage de complexité et de dépaysement qu'on peut le penser a priori. Il ne s'agit pas non plus de plaider pour un "patriotisme de l'ambiguité» identitaire $^{25}$, mais plutôt d'envisager la fragmentation des identités comme une potentialité. D'ailleurs, malgré sa détermination à démontrer la fausseté de l'opposition binaire entre les «static insider» que sont les autochtones observés et les «travelling outsider»» que représentent les ethnologues et anthropologues, James Clifford récuse toutes «nomadologies» :

My point, again, is not simply to invert the strategies of cultural localization, the making of "natives», which I criticized at the outset. I'm not saying there are no locales or homes, that everyone is - or should be - traveling, or cosmopolitan, or deterritorialized. This is not nomadology. Rather, what is at stake is a comparative cultural studies approach to specific bistories, tactics, everyday practices of dwelling and travelling: traveling-in-dwelling, dwelling-in-travelling. ${ }^{26}$

Cette dissémination identitaire rend donc impossible une éthique d'authenticité telle que définie dans la première partie. $\grave{A}$ l'inverse, la fragmentation contemporaine de l'identité fait en

24 Alain Touraine, Critique de la modernité, Paris, Fayard, 1992, p. 260.

25 Comme peut le faire par exemple Nancy Huston dans Pour un patriotisme de l'ambiguitté, Montréal, Fides, coll. "CÉTUQ», 1995, p. 38.

26 James Clifford, op. cit., p. 36. 
sorte qu'il y a autant de versions de l'authenticité québécoise qu'il y a de Québécois et de Québécoises, et ce, quelle que soit leur "souche». Le politique donne certes un contenu à la citoyenneté québécoise, mais l'authenticité, liée au domaine bien plus vaste de l'identité, ne peut pas être définie et confinée par un livre, une parole ou un discours. L'authenticité n'est pas «religieuse». Le "Je suis Québécois» prononcé serait donc le prédicat de l'authenticité québécoise. De plus, cette fragmentation de lidentité québécoise rend impossible la tentative de penser le Québec comme une communauté éthique d'inspiration hégélienne (Sittlichkeit), où la partie et le tout se réconcilient perpétuellement dans un ultime moment dialectique ${ }^{27}$. Comme nous le verrons, l'individu doit pouvoir entretenir un rapport agonique avec sa communauté. Or, ce constat n'a pas pour corollaire d'abandonner «l'idéal d'authenticité» décrit par Charles Taylor. On tentera de montrer que cet idéal moderne doit être repensé dans le cadre d'une éthique de créativité dont Foucault s'est fait le promoteur.

27 Voir Hegel, Principes de la philosophie du droit, Paris, Gallimard, 1995, pp. 189-377. 


\section{Foucault et l'éthique de créativitée 28}

Chez le Foucault de la fin des années 1970 et du début des années 1980 , l'omniprésence et la polysémie du pouvoir ainsi que la rétivité de la volonté sont deux pôles irréductibles et incontournables pour toute analyse de la subjectivité. Selon lui, les identités pratiques des sujets se construisent et sont construites au sein d'interactions agoniques entre les relations de pouvoir-savoir et les différents moyens de résistance déployés par le sujet. Le pouvoir et la résistance constituent l'un pour l'autre une condition de possibilité et un support nécessaire ${ }^{29}$. Or, malgré cette capacité de résistance conférée au sujet, il n'en demeure pas moins excessivement difficile pour le soi contemporain de résister aux fixations identitaires produites par une société normalisatrice / disciplinaire, un pouvoir spirituel, un bio-pouvoir, des technologies politiques ou par différents modes de "gouvemementalité». Comme le rappelle Foucault, le pouvoir s'exerce quotidiennement sur les individus en les «classant en catégories», en les désignant «par leur individualité propre», en les soumettant à une «loi de vérité» et en les

28 À première vue, il peut sembler paradoxal, voir incongru, de tenter de théoriser le sujet et son identité à l'aide de celui-là même qui a solennellement proclamé «la mort de l'Homme» et que d'aucuns considèrent comme le théoricien du pouvoir par excellence. Néanmoins, ce projet n'en est pas un que l'on doit mener contre Foucault. Dans la dernière phase de son travail, Foucault a affirmé en plus d'une occasion que le sujet et les "pratiques de liberté» constituaient les principaux thèmes de son travail intellectuel. Encore une fois, le présent essai ne me permet pas de justifier cette appropriation de Foucault. Voir aussi : James Tully, To think and act differently. Foucault contra Habermas, David Owen [éd.], Sage Publications, (à paraître) 1998.

29 Michel Foucault, «Le sujet et le pouvoin», Dits et écrits, vol. IV, Paris, Gallimard, 1994, p. 236. 
attachant «à leur identités ${ }^{30}$. Ce ne peut donc être que par une vigilance et une sensibilité extrêmes envers aujourd'bui que le sujet peut résister - tant bien que mal - aux «épinglements» identitaires susmentionnés. C'est dans ce contexte que l'approche généalogique adoptée par Foucault prend tout son sens. Les généalogies, en analysant le présent et en montrant la contingence de ce qui est donné comme nécessaire, peuvent permettre au sujet de défier les limites qui lui sont imposées et d'avoir ainsi la possibilité de penser différemment et de devenir autre. Aux dires de Foucault, ses généalogies sont "orientées vers "les limites actuelles du nécessaire" : c'est-à-dire vers ce qui n'est pas ou plus indispensable pour la constitution de nousmêmes comme sujets autonomes ${ }^{31 . » ~} D^{\prime}$ ailleurs, le rôle de l'intellectuel selon Foucault est précisément de dire ce qui est, en montrant comment cela pourrait être autrement.

C'est par une éthique de créativité inspirée par l'ethos de la modemité décrit dans l'article "Qu'est-ce que les Lumières ?̧» que Foucault considère que le sujet peut le mieux résister aux tentatives de menottement identitaire. Dans ce texte, Foucault avance que la modernité se caractérise mieux comme une

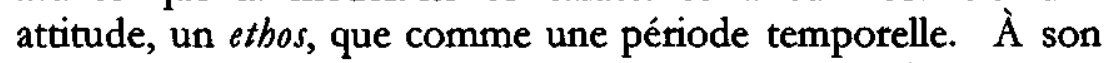
sens, Baudelaire est celui qui a capturé avec le plus d'acuité cette attitude typiquement moderne. Comme bien d'autres, le poète maudit a reconnu que la modemité était «autodestructive», c'està-dire dominée par la rupture, le mouvement perpétuel, le désordre, la mode et la nouveauté. Toutefois, Baudelaire situe l'ethos modeme dans le rapport que l'on adopte face à ce flux perpétuel; dans la capacité de saisir des éclats d'absolu dans un présent volatile :

30 Michel Foucault, «Le sujet et le pouvoin, loc. cit., p. 227. 31 Michel Foucault, "Qu'est-ce que les Lumières ?], Dits et écrits, vol. IV, p. 572. 
Pour lui, être moderne, ce n'est pas reconnaître et accepter ce mouvement perpétuel; c'est au contraire prendre une certaine attitude à l'égard de ce mouvement; et cette attitude volontaire, difficile, consiste à ressaisir quelque chose d'étemel qui n'est pas au-delà de l'instant présent, ni derrière lui, mais en lui. La modernité se distingue de la mode qui ne fait que suivte le cours du temps; c'est l'attitude qui permet de saisir ce qu'il y a «d'héroïque» dans le moment présent. La modernité n'est pas un fait de sensibilité au présent fugitif; c'est une volonté «d'héroïses» le présent ${ }^{32}$.

Être moderne, pour le poète maudit, implique de ne pas rester apathique et passif devant l'incertitude créée par une modemité autodestructrice. Il s'agit au contraire pour le sujet de se prendre comme un uobjet d'une élaboration complexe et dures et d'exercer sa liberté dans un présent fugitif. L'exercice de cette liberté, dans une perspective dandyste qui ressemble sensiblement au souci de soi gréco-romain (dans sa forme, et non dans sa substance), consiste en une critique permanente de nous-mêmes. Faire de soi une cuvre d'art, à recréer perpétuellement, au cœuur du règne moderne de l'éphémère : voilà le défi posé par Baudelaire - et Foucault - au sujet contemporain. Il s'agit là d'une éthique de créativité que le philosophe français oppose sans cesse à une éthique d'authenticité qui, de Platon aux psychanalystes contemporains, a dominé la pensée «humaniste». Plutôt que de découvrir une essence authentique, Foucault propose au sujet de se créer et de se recréer une identité qui se situe constamment sur la frontière entre le normal et l'anormal. Pour Foucault, l'identité n'est pas une découverte mais plutôt la possibilité de devenir autre chose que ce que l'on était au départ. En se faisant le promoteur d'une éthique de créativité, Foucault reprend à son compte le uself-

32 Ibid., p. 569. 
overcoming selfi nietzschéen. Le sujet nietzschéen, privé de repères moraux et de vérités universelles pour contraindre/orienter ses actions, se retrouve quotidiennement devant la responsabilité de transformer et de dépasser sa situation actuelle. Dans un contexte où le sujet doit péniblement se définir au coeur de relations de pouvoir qui tentent de l'attacher à certaines identités, Foucault considère que la subjectivité ne tient qu'à la capacité de se remanier continuellement afin de dénouer les identités trop étouffantes. Pour éviter «l'épinglement identitaire», le sujet ne peut que se questionner sans relâche, se problématiser et parfois se recomposer. Bref, comme le suggère Jean Larose en toute contiguité d'esprit, l'identité peut être envisagée comme «une éternelle mise en jeu ${ }^{33} . »$

L'éthique de créativité proposée par Foucault ne prescrit pas au sujet la façon de définir substantivement son identité. Pour Foucault, ce n'est pas le contenu des différents fragments identitaires du sujet qui possède une valeur esthétique, mais plutôt le processus de création/recréation; l'ethos dandyste qui consiste à se remettre soi-même cent fois sur le métier. Toujours dans une perspective nietzschéenne, Foucault considère comme «belle» une vie "d'auto-problématisation» constante. À ses yeux, la capacité de résister (toujours partiellement et jamais définitivement) à l'hétéronomie n'est pas une tâche purement négative; c'est aussi un acte de création. Ce qui donne de la valeur à la vie, au travail et à l'amour, selon Foucault, c'est qu'ils permettent au sujet de développer une "curiosité», un sens aiguisé du réel qui lui permet ensuite d'explorer de nouvelles formes de subjectivité et d'échapper à l'embrigadement identitaire. Bref, comme le rappelle David Owen, la dignité du sujet ne réside pas dans ce qu'il est

33 Dans Marcos Ancelovici et Francis Dupuis-Déri, op. cit., p. 73. 
(fatalement et authentiquement), mais au contraire dans sa capacité de décliner (créativement) son identité au pluriel ${ }^{34}$.

Avec cette esthétique de l'existence, où le sujet fait de sa vie une cuvre d'art, Foucault tente de dissocier subjectivité et authenticité. Par exemple, il reproche à Sartre (plutôt injustement, d'ailleurs) l'introduction d'une notion morale d'authenticité dans une philosophie existentialiste qui a pourtant proclamé que «l'existence précède l'essences) ${ }^{35}$. Le professeur au Collège de France s'en prend également aux thérapies psychanalytiques qui postulent l'existence d'un moi immaculé, bien enfoui dans les profondeurs de l'inconscient, qu'il s'agirait de défricher et de «libéres» du joug réprobateur du surmoi. Malgré ces doutes légitimes, il convient de se demander si l'empressement de Foucault de se débarrasser de cette notion d'authenticité est bel et bien nécessaire. Ainsi qu'on l'avançait plus tôt, le contemporain, pris dans une modernité fugitive, tente de se référer à certains repères qui hui permettent de garder le cap dans l'élaboration difficile de ses trajectoires identitaires. Ces repères, dans un univers désenchanté, ne peuvent toutefois plus être transcendantaux. Ils ne peuvent qu'émaner de la subjectivité et/ou du contexte social du sujet. Il n'en demeure pas moins que le soi contemporain peut vouloir rester fidèle à certains repères immanents et subjectifs qui hii permettent de s'orienter dans un monde nietzschéen. La notion d'authenticité peut jouer ce rôle de uboussole» subjective permettant au sujet de garder une certaine cohérence dans l'écriture de son récit identitaire. Comme le rappelle Charles Taylor, l'authenticité est un idéal moral qui s'est développé en concomitance avec la

34 David Owen, Maturity and Modernity; Nietzsche, Weber, Foucault and the ambivalance of reason, London and New York, Routledge, 1994, p. 297.

35 Michel Foucault, «À propos de la généalogie de l'éthique : un aperçu du travail en cours", Dits et écrits, vol. IV, loc. cit., PP. 392393. 
conversion de l'identité en un projet narratif. De toute évidence, l'éthique de créativité proposée ici est incompatible avec lidéal d'authenticité proposé par les théoriciens du sentiment moral, Rousseau ou Herder. Pour eux, la personne authentique est celle qui demeure fidèle à une voix, une essence ou une intuition intérieure. Toutefois, en prétendant que l'authenticité demeure un idéal valable, Taylor ne fait pas référence à une sincérité que l'individu devrait manifester envers une essence intérieure découverte en restant à l'écoute de soi-même. Selon le philosophe montréalais, l'idéal d'authenticité implique également une part de création et d'opposition aux règles sociales : «schématiquement, on peut dire que l'authenticité implique $(i)$ une création et une construction aussi bien qu'une découverte, (ii) une originalité, (iii) et souvent une opposition aux règles sociales et même, éventuellement, à ce que nous reconnaissons comme la morale ${ }^{36} . 川$ Pour Taylor, l'idéal d'authenticité engage le contemporain autant à découvrir qu'à formuler son identité. En conséquence, lidéal d'authenticité contemporain n'est pas exactement la quête spirituelle que Foucault aimait décrire et critiquer.

Malgré cela, la seule façon d'intégrer l'authenticité à une éthique de créativité est peut-être de faire de cet idéal une construction plutôt que le produit d'un processus de création / découverte. Bien qu'il n'en soit pas ainsi chez le philosophe montréalais ${ }^{37}$, le danger que représente la notion d'authenticité

36 Charles Taylor, Grandeur et misère de la modernité, Montréal, Bellarmin, 1992, p. 86.

$37 \mathrm{La}$ "découverte» et «'authenticité» évoquées par Taylor semblent parfois se référer à l'exploration que le sujet doit effectuer au sein de sa propre culture afin de se réaliser comme un agent humain à part entière, alors qu'elles semblent en d'autres occasions concemer les ustrong evaluations), c'est-à-dire les profondes évaluations de soi qui sont à la base du processus de formation identitaire. Dans les deux cas, cette découverte d'une authenticité individuelle a peu à voir 
est qu'elle soit associée à une essence pure et statique que l'individu doit découvrir en lui-même ou à l'intérieur de sa communauté. Or, c'est marement le cas. Comme le rappelle encore une fois James Clifford, l'authenticité est une «inventive impuritys; un amalgame idiosyncratique composé d'éléments puisés dans la multiplicité de filières identificatrices accessibles aux individus. Les sources de l'authenticité sont plurielles, éclatées et parfois même, contradictoires. L'authenticité émerge donc de la complexité plutôt que la pureté ou de la staticité. " "Authenticity" is seldom an all-or-nothing issuen, affirme d'ailleurs Clifford à cet effet ${ }^{38}$. Remaniée, l'authenticité hybride ou plurielle fait donc référence à une création identitaire à laquelle le sujet tente de rester fidèle afin de se retrouver dans les dédales (post)modernes de l'identité contemporaine. De cette facon, l'authenticité (hybride / plurielle), perçue comme un référent identitaire acquis et construit par le sujet, peut demeurer un idéal valable même à l'intérieur d'une éthique de créativité.

Retranscrite dans le récit identitaire québécois, cette éthique de créativité disqualifie toute définition substantive de l'identité. Lidentité est vue comme une question ouverte, où chaque réponse trouvée peut être problématisée, révisée et peut-être corrigée. Toutefois, malgré la croyance populaire, penser le processus de formation de l'identité individuelle dans les termes de l'éthique de créativité foucaldienne ne mène ni au cosmopolitisme ni à un individualisme libéral de type rawlsien. Au contraire, la langue et la communauté sont, comme l'ont montré les analyses de Charles Taylor, des horizons identitaires

avec les critiques de Foucault mentionnées précédemment. Voir Charles Taylor, "L'atomisme», La Liberté des modernes, Paris, PUF, 1997, p. 252. et "What is human agency?», Human Agency and Language, Philosophical Papers 1, Cambridge University Press, 1985, p. 38 .

38 James Clifford, Routes, op. cit., p. 178. 
incontournables pour le sujet contemporain ${ }^{39}$. De plus, l'individu ne peut développer une éthique de créativité, qui lui permettra de dénouer les nouds identitaires qui se sont formés dans sa vie, qu'en interagissant au sein de sa communauté avec des «autruis donneurs de sens». L'usage de droits collectifs, ayant par exemple pour but la promotion d'une langue et d'une culture, se trouve ainsi justifié au nom d'une esthétique d'existence. Même le philosophe Will Kymlicka, dont la conception instrumentale de la communauté demeure problématique, admet que l'ontologie libérale a toujours failli à reconnaitre l'importance de la langue et de la communauté dans la formation de l'identité moderne : «liberals, in a misguided attempt to promote the dignity and autonomy of the individual, have undermined the very communities and associations which alone can nurture buman flourishing and freedom.» ${ }^{40}$

Il faut par conséquent cesser de voir la nation, qui est la plus puissante source d'identification collective de l'individu moderne, uniquement comme un lieu d'incarcération identitaire ${ }^{41}$. Si l'on doit avec raison considérer la nation comme

39 Voir par exemple Charles Taylor, «Pourquoi les nations doiventelles se transformer en Étab, Rapprocher les solitudes. Écrits sur le fédéralisme et le nationalisme au Canada, Sainte-Foy, Presses de l'université Laval, 1992, p. 53.

${ }^{40}$ Will Kymlicka, Liberalism, community and culture, Oxford, Clarendon Press, 1989, p. 2.

41 Foucault lui-même semble parfois partager une vision purement négative de la communauté et de la nation. Voir par exemple «La technologie politique des individus", Dits et écrits, vol. IV, loc. cit., p. 814. Toutefois, Foucault reconnaît que le sujet élabore ses pratiques de liberté en évoluant au sein d'un réseau de conduites préétablies, dans un champ de possibles qui lui est offert /imposé par sa situation sociale, culturelle, politique et économique : «Je pense au contraire que le sujet se constitue à travers des pratiques d'assujetissement, ou, liberté comme, dans l'Antiquité, à partir, bien entendu, d'un certain nombre de règles, styles, conventions, qu'on retrouve dans le milieu culturel.» C'est ainsi que des politiques visant 
source d'oublis, d'illusions et de mythes, on doit aussi la considérer comme productrice de lucidité et d'originalité (puisque les capacités de résistance du sujet n'émergent pas d'un vacuum). L'identification et l'appartenance à une communauté nationale peuvent être une condition de possibilité pour la résistance du sujet contemporain. Comme le note Alain Touraine, le sujet ne peut résister aux forces de nomalisation qu'en tenant ses divers fragments de vie dans une tension perpétuelle :

Le sujet associe le plaisir de vivre à la volonté d'entreprendre, la diversité des expériences vécues au sérieux de la mémoire et de l'engagement. Il a besoin que le Ça rompe les défenses du sumoi autant que d'être fidèle à un visage ou à une langue; parce que la force du désir comme celle de la tradition, l'appel de la consommation et du voyage autant que celui de la recherche et de la production libèrent des rôles et des nomes qu'imposent les systèmes et qui objectivent le sujet pour mieux le contrôler't2.

L'antinationalisme se trouve donc affligé par le mal qu'il dénonce : «l'épinglement» de l'identité, pour reprendre les mots de Régine Robin. En effet, en bloquant l'accès à une identification collective commune, les antinationalistes figent les Québécois dans une identité cosmopolite qui fragilise le cmilieu culturel» dans lequel une éthique de créativité peut être élaborée. Par conséquent, si l'on prend au sérieux l'entreprise d'élaborer une théorie formelle de l'identité qui refuse toute forme de fixation et de hiérarchisation des différents fragments

à protéger et à promouvoir le «milieu cultureb» peuvent être légitimées dans les termes d'une éthique de créativité. Michel Foucault, "Une esthétique de l'existence», op. cit., p. 733.

42 Alain Touraine, Critique de la modernité, op. cit., p. 284. 
identitaires, l'on ne peut que mettre dos à dos les nationalismes et les cosmopolitismes uexclusivistes». D'ailleurs, des auteurs comme Clifford et Todorov sont à la fois nationalistes et antinationalistes; cosmopolitains et anticosmopolitains. En d'autres termes, les deux penseurs s'opposent à toute détermination hétéronome et paternaliste de lidentité du sujet contemporain. Le contemporain peut être à la fois arpenteur et navigateur, pour reprendre la symbolisation de Monique LaRue. Actif, le sujet fait éclater ses réseaux d'appartenance et offre, au conditionnel, sa loyauté à des allégeances diverses et parfois même contradictoires. Angoissé, il se garde bien de rompre avec ses filiations identitaires traditionnelles, telles sa langue, sa communauté et ses "romans mémoriels», qui sont aussi sources de confort et de richesse.

\section{Entre le confort et l'indifférence, l'identité québécoise}

En terminant ces prolégomènes, qui se veulent une contribution à une entreprise déjà amorcée de théorisation formelle - plutôt que substantive - de l'identité québécoise, il est important de rappeler certaines propositions. D'abord, la recherche, par un courant important du nationalisme québécois d'une authenticité présumément perdue, apparaît hautement problématique. Le dépaysement et la fragmentation identitaire qui sont susceptibles d'habiter le Québécois contemporain, quelles que soient sa langue, sa "souche», sa vision du passé ou ses allégeances politiques, font en sorte que toute définition substantive de la québécitude risque fort de disqualifier chaque Québécois pris individuellement. Il ne s'agit donc plus de capturer l'être québécois, mais plutôt d'explorer le devenir québécois. Ensuite, il est tout aussi important de rappeler que l'antinationalisme brandi par les critiques séculaires du nationalisme québécois s'avère tout autant incompatible avec 
une tentative de théorisation formelle qui fait de l'identité une question ouverte. L'identité québécoise doit permettre l'enracinement autant que l'arrachement. En refusant l'identification à certaines valeurs convergentes, les antinationalistes «épinglent» à leur façon l'identité des Québécois(es). L'identité québécoise contemporaine est composée d'éléments mémoriels et civiques, historiques et constitutionnels, temporels et spatiaux, imaginaires et matériels, locaux et mondiaux et toute tentative d'homogénéisation ou de purification de ladite identité - dans un sens ou dans l'autre heurte de plein fouet la possibilité pour le Québécois de décliner son identité au pluriel. Ce demier, attaché à des valeurs qu'il puise parfois dans une vision du passé, parfois d'une projection dans l'avenir, n'a pas envie de tarir l'une ou l'autre des sources de son identité. Bref, entre le confort d'une authenticité tricotée serrée et l'indifférence à l'égard de valeurs communes qu'une majorité de Québécois(es) ressent le besoin d'affirmer, une identité québécoise plurielle et labile tente laborieusement de se profiler.

Donc, même s'il devient de plus en plus difficile d'élaborer des raisons communes sur lesquelles pourrait être basée la société québécoise de demain, l'une d'entre elles se doit d'être la reconnaissance que la québécitude est une création qui s'exprime de façon polymorphe, et que l'authenticité québécoise est métissée et sans cesse évolutive. La fluidité et la porosité des frontières de l'identité doivent être prises au sérieux. Concrètement, cette reconnaissance signifie que les nationalistes québécois «républicains» acceptent que certains de leurs compatriotes considèrent la Conquête comme un événement historique parmi d'autres plutôt que comme un traumatisme, et refusent en conséquence de faire de la vindicte historique l'essence de leur identité, et de la «rhétorique de l'humiliation» 
leur mode d'expression ${ }^{43}$. Cela signifie aussi que les Québécois acceptent que les communautés autochtones vivant sur le territoire du Québec se pensent comme peuples autonomes, considèrent leur attachement au Québec comme étant instrumental, et insistent pour que toute négociation politique bilatérale se fonde sur une relation de nation à nation. Finalement, la reconnaissance du fait qu'il existe plusieurs manières d'être Québécois(e) implique également que l'on ne demande pas à la partie de la population qui s'identifie à une langue, à une vision de l'histoire et à une projection dans l'avenir sensiblement communes de renoncer à leur version de la québécitude. Cette acceptation générale et mutuelle qu'il existe une pluralité d'authenticités québécoises procurerait à tous les Québécois la reconnaissance nécessaire au maintien d'une identité personnelle qui ne peut plus se voir confirmée par des sources métanarratives ou transcendantales ${ }^{44}$. Inévitablement, ces visions de l'identité québécoise s'entrechoqueront, et c'est dans l'agora que doit être élaborée, de façon agonique plutôt qu'antagonistique, l'identité collective du Québec contemporain. C'est de la délibération et non des manuels d'histoire ou d'un historicisme cosmopolitique, que doit émaner la spécificité de la québécitude. Or, le politique, tant au Québec qu'au Canada, n'a pas encore relevé le défi de l'indétermination identitaire contemporaine. Ni le Canada de 1982, qui refuse obstinément de se penser comme une fédération multinationale, ni le projet souverainiste traditionnel, qui n'a pas encore prouvé qu'il

43 Comme le suggère par exemple : Guy Laforest, «Dépasser le ressentiment), De l'urgence. Textes politiques 1994-1995, Montréal, Boréal, 1995, pp. 167-169. Pour un exemple de vindicte historique et de rhétorique de l'humiliation, voir : Josée Legault, "Comme si le Québec vivait une seconde conquête», Le Devoir, 25 août 1998, p. A7.

44 Charles Taylor, Multiculturalisme. Différence et démocratie, op. cit., p. 53. 
pouvait intégrer l'hétérogène ${ }^{45}$, n'ont jusqu'ici pris au sérieux la complexité de lidentité québécoise. Une véritable fédération multinationale, telle que celle qui est revendiquée par une majorité de Québécois et d'Autochtones, et édifiée sur la base de la reconnaissance et du respect mutuels, pourrait être un cadre politique propice à l'épanouissement d'identités plurielles. Par définition, le fédéralisme multinational favorise la superposition des identités. Or, comme l'affirme Will Kymlicka, le modèle plurinational compte peu d'appuis dans le "Canada-horsQuébec ${ }^{45}$. La vision trudeauiste du Canada comme une grande nation d'un océan à l'autre est toujours celle qui domine dans les esprits. Dans la même veine, le "républicanisme» québécois n'a pas encore démontré qu'il consentait à accepter et valoriser la pluralité des appartenances et des loyautés ${ }^{47}$. Encore aujourd'hui, on demande d'un côté comme de l'autre aux Québécois de renoncer à l'une de leurs filières identificatrices; ce qu'ils refusent régulièrement depuis près de trente ans. Bref, le politique n'a pas encore fait preuve de la modestie et du labeur qui sont de mise lorsqu'il s'agit de traiter avec les pérégrinations contemporaines de l'identité.

45 Voir Daniel Salée, «La mondialisation et la construction de l'identité au Québec"), Les Frontières de l'identité, op. cit., pp. 110-123. 46 Will Kymlicka, «Le fédéralisme multinational au Canada : un partenariat à repensen,, Sortir de l'impasse. Les voies de la réconciliation, sous la direction de Guy Laforest et Roger Gibbins, Montréal, I.R.P.P., 1998, pp. 15-54.

47 Plutôt que de s'inspirer du modèle de la république française, le projet de souveraineté-partenariat québécois doit par exemple donner l'assurance aux membres la communauté anglophone qu'il leur serait toujours possible de s'identifier d'abord au Canada, et aux peuples autochtones à leur communauté d'origine, même dans l'éventualité d'un Québec souverain (mais partenaire). Le Québec parviendrait peut-être ainsi à contoumer les apories qui ébranlent le Canada eversion Trudeau». 